\title{
Intracellular calcium increase and acrosome reaction in response to progesterone in human spermatozoa are correlated with in-vitro fertilization
}

\author{
Csilla Krausz, Lorella Bonaccorsi, Michaela Luconi, \\ Beatrice Fuzzi ${ }^{1}$, Luciana Criscuoli ${ }^{1}$, \\ Sandra Pellegrini ${ }^{1}$, Gianni Forti and \\ Elisabetta Baldi ${ }^{2}$ \\ Dipartimento di Fisiopatologia Clinica, Unita di Andrologidà and \\ ${ }^{1}$ Clinica Ostetrica e Ginecologica, Università di Firenze, viale \\ Piericcini, 6-50139 Firenze, Italy \\ ${ }^{2}$ To whom correspondence should be addressed
}

In this study we have investigated responsiveness to progesterone in spermatozoa from a group of unselected male partners of couples undergoing in-vitro fertilization (IVF). We evaluated progesterone-stimulated intracellular $\mathrm{Ca}^{2+}$ $\left(\left[\mathrm{Ca}^{2+}\right]_{\mathrm{i}}\right)$ and percentage increase in acrosome reaction in the same sperm sample used for oocyte inseminations. $\left[\mathrm{Ca}^{2+}\right]_{i}$ was measured with a fluorimetric method, while the acrosome reaction was assessed using a fluorescent probe (fluorescein isothiocyanate-labelled peanut lectin). The average percentage $\left[\mathrm{Ca}^{2+}\right]_{i}$ as well as the rate of increase in the frequency of acrosome reaction following progesterone challenge were significantly lower $(P<\mathbf{0 . 0 0 5})$ in the group of patients with a fertilization rate $<50 \%$. In addition, significant correlations between the fertilization rate and the progesterone-stimulated $\left[\mathrm{Ca}^{2+}\right]_{\mathrm{i}}$ and acrosome reaction increases $(r=0.78$ and $r=$ 0.79 respectively) were observed. Furthermore, in cases of fertilization failure, no increase of $\left[\mathrm{Ca}^{2+}\right]_{i}$ or acrosome reaction was observed in response to progesterone with the exception of one case. Our results indicate that $\left[\mathrm{Ca}^{2+}\right]_{i}$ and acrosome reaction increases in response to progesterone can be of value in the prediction of sperm fertilizing ability. As the two parameters were significantly correlated to each other $(r=0.86)$, the two assays have similar IVF predictive value and might be used interchangeably as a diagnostic tool in the assignment of male patients to the different kinds of assisted fertilization techniques.

Key words: acrosome reaction/intracellular calcium/in-vitro fertilization/progesterone

\section{Introduction}

Although in-vitro fertilization (IVF) is one of the most effective treatments of human infertility, failure of oocyte fertilization is quite a frequent outcome in routine IVF treatment, especially in cases of male infertility (Winston and Handyside, 1993). The possibility of predicting the fertilizing ability of human spermatozoa would thus be of great value considering the human involvement and the high cost of the IVF technique. In the past few years several studies have been performed to find a good predictive parameter of sperm fertilizing ability (Liu and Baker, 1992; Winston and Handyside, 1993). With the exception of morphology assessed with strict criteria, traditional parameters of semen analysis, such as concentration, motility and viability of spermatozoa, have little or no predictive value for sperm fertilizing ability (Liu and Baker, 1992). It is generally believed that biochemical alterations of sperm function are responsible for defective sperm-egg interaction and lack of fertilization. An alteration of sperm capacity to undergo acrosome reaction in response to ionophores has been shown in human spermatozoa with low fertilizing ability (Aitken et al., 1987; Pampiglione et al., 1993). However, such studies were performed with a nonphysiological stimulus of acrosomal exocytosis. Progesterone has been indicated recently as a physiological stimulus of acrosome reaction in human spermatozoa (Osman et al., 1989; Baldi et al., 1991; Meizel and Turner, 1991) and appears to be primarily involved in the action of follicular fluid on spermatozoa, as the ability of the latter to stimulate acrosome reaction is highly correlated with its progesterone content (Morales et al., 1992; Saaranen et al., 1993). A decreased sperm responsiveness to progesterone has been shown recently in oligozoospermic (Falsetti et $a l ., 1993$ ) as well as in some infertile patients (Tesarik and Mendoza, 1992), suggesting an alteration of the putative sperm membrane progesterone receptor function in some cases of male infertility. In addition, a recent study demonstrated that progesterone-induced increase of sperm $\left[\mathrm{Ca}^{2+}\right]_{i}$ is significantly correlated with egg-penetrating ability of spermatozoa from fertile but not infertile men (Shimizu et al., 1993), although a weak tendency for these two phenomena to be correlated was also observed for infertile men (Shimizu et al., 1993). It must be noted, however, that this study was performed with zona-free hamster egg and thus under conditions quite different from the physiological one.

This study was conducted in spermatozoa from unselected men undergoing IVF, to investigate if the ability of spermatozoa to fertilize human oocytes in vitro was related to the responsiveness of spermatozoa (measured as increase of $\left[\mathrm{Ca}^{2+}\right]_{i}$ and acrosome reaction) to progesterone.

Materials and methods

\section{Chemicals}

Fura 2/AM and ionomycin were obtained from Calbiochem (San Diego, CA, USA). Progesterone and fluorescein isothiocyanate (FITC)-labelled lectin were obtained from Sigma (St Louis, MO, USA). IVF medium was obtained from MediCult (Denmark). 
Progesterone was dissolved in dimethylsulphoxide (DMSO) at an initial concentration of $10 \mathrm{mg} / \mathrm{ml}$ and further dilutions were made in IVF medium.

\section{Subjects}

The study population consists of 22 unselected couples undergoing IVF therapy. Nine couples were affected by pure female factor infertility, six by male factor infertility and three by both tubal and male factor. In four cases the infertility was of unknown aetiology. Measurement of $\left[\mathrm{Ca}^{2+}\right]_{i}$ was performed in 19 subjects and acrosome reaction was assessed in 16 subjects.

\section{Preparation of spermatozoa}

Spermatozoa were washed from seminal plasma; motile spermatozoa were separated by the swim-up procedure in IVF medium, washed and divided into two aliquots, one for subsequent oocyte insemination and the other for calcium and acrosome reaction studies. Oocytes were collected under transvaginal ultrasound guidance from women previously treated according to the following protocol: s.c. buserelin acetate (Suprefact; Hoechst, Italia, Scoppitto, Italy; $600 \mu \mathrm{g} / \mathrm{day}$ ) starting from day 21 of the last menstrual cycle to desensitize the pituitary gland. When pituitary desensitization has been achieved, as shown by a concentration of serum oestradiol of $<50 \mathrm{pg} / \mathrm{ml}$, buserelin acetate treatment was continued together with purified folliclestimulating hormone (FSH; 225 IU/day, Metrodin, Serono, Rome, Italy). Ovarian stimulation was monitored by daily ultrasound assessment of follicular growth and estimation of serum oestradiol concentration. When serum oestradiol was $>600 \mathrm{pg} / \mathrm{ml}$, women were treated with $10000 \mathrm{IU}$ human chorionic gonadotrophin (HCG, Profasi HP; Serono) and oocyte retrieval was performed $34 \mathrm{~h}$ later. An average of $7.60 \pm 0.94$ ova per woman was recovered during this procedure.

\section{Measurement of $\left[\mathrm{Ca}^{2+}\right]_{i}$}

After the swim-up procedure, spermatozoa were resuspended at a concentration of $5 \times 10^{6} / \mathrm{ml}$ in IVF medium, incubated with fura $2 / \mathrm{AM}$ for $45 \mathrm{~min}$ and $\left[\mathrm{Ca}^{2+}\right]_{i}$ measured as described previously using a spectrofluorimetric method (Baldi et al., 1991; Falsetti et al., 1993). Spermatozoa were stimulated with 0.1 and $1.0 \mu \mathrm{g} / \mathrm{ml}$ progesterone. Fluorescence measurements were converted to $\left[\mathrm{Ca}^{2+}\right]_{\mathrm{i}}$ by determining maximal fluorescence $\left(F_{\max }\right)$ with ionomycin ( $8 \mathrm{mM}$ final concentration) followed by minimal fluorescence $\left(F_{\min }\right)$ with $10 \mathrm{mM}$ ethylene glycol tetraacetic acid (EGTA), pH 10. $\left[\mathrm{Ca}^{2+}\right]_{i}$ was calculated according to Grynkiewicz et al. (1985) assuming a dissociation constant of fura 2 for calcium of $224 \mathrm{nM}$. The addition of ionomycin yielded similar increases in fluorescence in all the samples examined, indicating a similar fura 2/AM incorporation. Autofluorescence of the cells was assessed by measuring the fluorescence of unloaded cells; autofluorescence subtraction did not modify basal or stimulated $\left[\mathrm{Ca}^{2+}\right]_{\mathrm{i}}$, as reported previously (Falsetti et al., 1993).

\section{Determination of acrosome reaction}

Acrosome-reacted spermatozoa were evaluated using the fluorescent probe FITC-labelled Arachis hypogaea (peanut) lectin, as described previously (Aitken et al., 1993; Krausz et al., 1994). Briefly, spermatozoa recovered after the swim-up procedure were incubated with progesterone $(4 \mu \mathrm{g} / \mathrm{ml})$ or DMSO control solvent for $1 \mathrm{~h}$ at $37^{\circ} \mathrm{C}$, centrifuged and further incubated in $0.5 \mathrm{ml}$ of hypo-osmotic swelling medium for $1 \mathrm{~h}$. After centrifugation, spermatozoa were finally suspended in $50 \mu \mathrm{l}$ of ice-cold methanol, layered on a slide, stained with fluorescent lectin and scored using a fluorescence microscope. The acrosome reaction was evaluated in a total of 100 spermatozoa/slide. Only curly tailed spermatozoa were scored. Control solvent gave identical results to those given by IVF medium alone.

\section{Statistical analysis}

Results are expressed as mean \pm SEM. Statistical comparisons were performed using the non-parametric Wilcoxon test for paired and unpaired data. Correlation analyses were performed using Pearson's correlation coefficient.

\section{Results}

\section{Basal and progesterone-stimulated sperm $\left[\mathrm{Ca}^{2+}\right]_{i}$}

Subjects were arbitrarily divided into two groups on the basis of percentage fertilization rate (\%FR): group 1 with a \%FR $\geq 50 \%$ (average $\% \mathrm{FR}, 71.3 \pm 4.4, n=9$ ) and group 2 with a $\%$ FR $<50 \%$ (average $\% F R, 13.7 \pm 4.6, n=13$ ). In group 2 , spermatozoa from seven subjects which did not fertilize any oocyte were also included. Concentration and motility of spermatozoa in seminal fluid and after the swim-up procedure in the two groups of patients are reported in Table I. As shown in the table, basal and post-swim-up sperm parameters were significantly different in the two groups; however, poor correlation with \%FR was observed for all parameters $(r=0.48$, $P<0.05$ and $r=0.32$, not significant, respectively, for preand post-swim-up sperm number, and $r=0.50, P<0.05$ and $r=0.54, P<0.01$, respectively, for pre- and post-swim-up sperm motility). Moreover, in the seven patients whose spermatozoa failed to fertilize, sperm motility ranged from 5 to $50 \%$ in seminal fluid and from 50 to $90 \%$ after the swim-up

Table I. Concentration and motility of spermatozoa in seminal fluid and after swim-up in the two groups of subjects

\begin{tabular}{|c|c|c|c|c|c|}
\hline Group & $\% \mathrm{FR}$ & $\begin{array}{l}\text { Spermatozoa }\left(\times 10^{6} / \mathrm{ml}\right) \\
\text { in seminal fluid }\end{array}$ & $\begin{array}{l}\text { Total motility (\%) } \\
\text { in seminal fluid }\end{array}$ & $\begin{array}{l}\text { Spermatozoa }\left(\times 10^{6} / \mathrm{ml}\right) \\
\text { after swim-up }\end{array}$ & $\begin{array}{l}\text { Total motility (\%) } \\
\text { after swim-up }\end{array}$ \\
\hline $\begin{array}{l}1 \\
2\end{array}$ & $\begin{array}{l}\geq 50(n=9) \\
<50(n=13)\end{array}$ & $\begin{aligned} 134.2 & \pm 27.9 \\
57.6 & \pm 10.2^{\mathrm{a}}\end{aligned}$ & $\begin{array}{l}53.8 \pm 5.6 \\
33.8 \pm 3.3^{\mathrm{a}}\end{array}$ & $\begin{aligned} 15.1 & \pm 3.5 \\
7.6 & \pm 2.1^{\mathrm{a}}\end{aligned}$ & $\begin{array}{l}86.1 \pm 4.7 \\
75.0 \pm 3.4^{a}\end{array}$ \\
\hline
\end{tabular}

FR = fertilization rate.

${ }^{\mathrm{a}} P<0.05$. 
procedure, indicating poor predictive value of this parameter with respect to IVF outcome.

Average basal $\left[\mathrm{Ca}^{2+}\right]_{\mathrm{i}}$ in fura 2-loaded spermatozoa from our subjects was $188.3 \pm 18.8 \mathrm{nM}(n=19)$; there was no difference in basal $\left[\mathrm{Ca}^{2+}\right]_{\mathrm{i}}$ between the two groups of subjects $(174.7 \pm$ 26.0 and $235 \pm 30 \mathrm{nM}$ in groups 1 and 2 respectively). $\left[\mathrm{Ca}^{2+}\right]_{\mathrm{i}}$ increase in response to both 0.1 and $1.0 \mu \mathrm{g} / \mathrm{ml}$ progesterone was significantly higher in subjects with a FR $\geq 50 \%$ compared with subjects with a $<50 \%$ FR (Figure 1); the significance between the two groups was more evident when $0.1 \mu \mathrm{g} / \mathrm{ml}$ progesterone was used to stimulate spermatozoa $(3.2 \pm 0.4$-fold increase in group 1 compared with $1.4 \pm 0.2$-fold increase in group $2, P$ $<0.005)$. Similar statistical differences between \%FR were obtained when the subjects were divided on the basis of

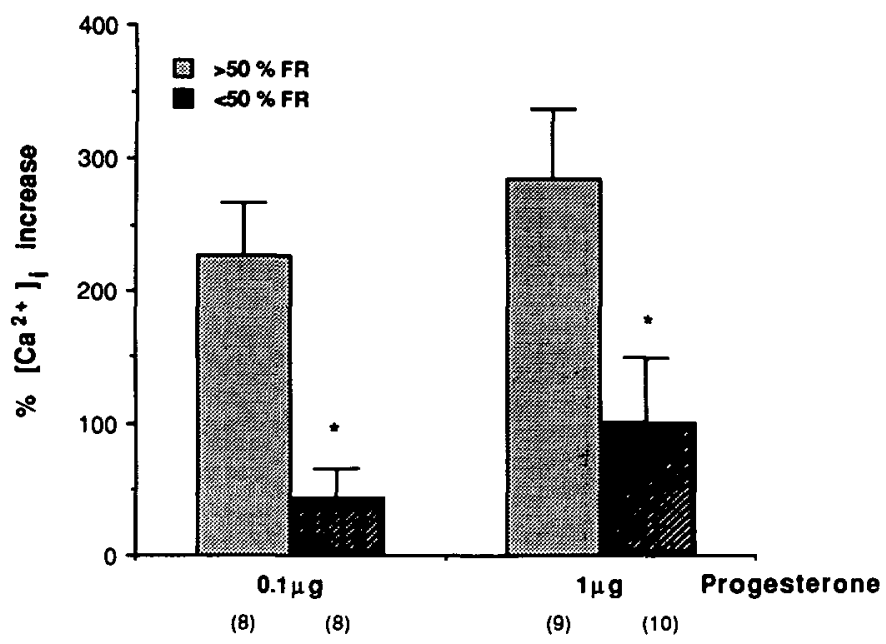

Fig. 1. Percentage increase of sperm $\left[\mathrm{Ca}^{2+}\right]_{i}$ following 0.1 and 1.0 $\mu \mathrm{g} / \mathrm{ml}$ progesterone challenge in patients with a fertilization rate (FR) $\geq 50$ and $<50 \%$. Numbers of patients shown in parentheses. $P<0.005$ versus $\geq 50 \%$ FR.

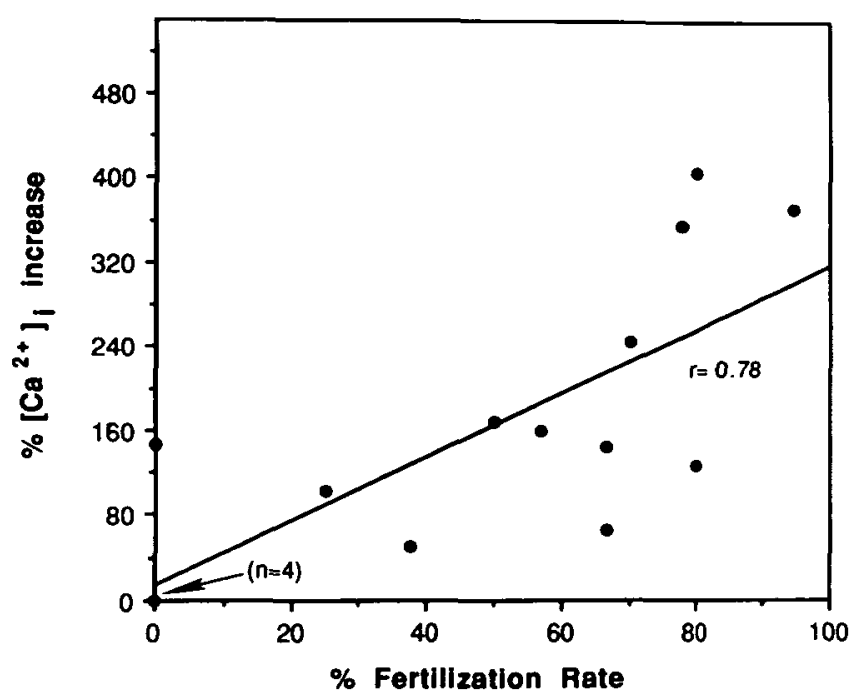

Fig. 2. Correlation curve between sperm percentage fertilization rate and percentage increase of sperm $\left[\mathrm{Ca}^{2+}\right]_{i}$ following challenge with $0.1 \mu \mathrm{g} / \mathrm{ml}$ progesterone in the subjects included in our study. The number of patients with zero $\left[\mathrm{Ca}^{2+}\right]_{i}$ increase and fertilization failure is shown in parentheses. progesterone-stimulated $\left[\mathrm{Ca}^{2+}\right]_{\mathrm{i}}$ increase (data not shown). It is worth while noting that in four out of the five subjects with lack of fertilization, no $\left[\mathrm{Ca}^{2+}\right]_{i}$ increase was observed in response to either progesterone concentrations. The $\left[\mathrm{Ca}^{2+}\right]_{i}$ increase in response to both 0.1 and $1.0 \mu \mathrm{g} / \mathrm{ml}$ progesterone was significantly correlated to \%FR $[r=0.78, P<0.001$ for $0.1 \mu \mathrm{g} / \mathrm{ml}$ progesterone (Figure 2) and $r=0.69, P<0.001$ for $1.0 \mu \mathrm{g} / \mathrm{ml}$ progesterone (data not shown)].

\section{Basal and progesterone-stimulated acrosome reaction in the two groups of subjects}

Basal percentage acrosome reaction was not different in the two groups of subjects (Figure 3), whereas a significant difference was present after stimulation with $4 \mu \mathrm{g} / \mathrm{ml}$ progesterone (Figure 3 ). The difference between percentage progesterone-induced and percentage spontaneous acrosome-reacted spermatozoa, considered to be the percentage of spermatozoa in the population capable of responding to progesterone [and thus called acrosome reaction following progesterone challenge (ARPC)], was significantly correlated to \% FR $(r=0.79, P<0.001$; Figure 4a). Moreover, the ARPC was significantly correlated to $\left[\mathrm{Ca}^{2+}\right]_{\mathrm{i}}$ increase in response to $0.1 \mu \mathrm{g} / \mathrm{ml}$ progesterone $(r=$ $0.86, P<0.001, n=11$; Figure $4 \mathrm{~b}$ ), indicating a strict dependence between the two parameters. In three out of four subjects with fertilization failure where acrosome reaction in response to progesterone was evaluated, no response was observed, whereas in one subject we found an ARPC of $5 \%$.

\section{Discussion}

Male infertility accounts for $\sim 25 \%$ of all human infertility and IVF appears to be, at the moment, the only effective treatment. However, lack of fertilization is quite frequent in cases of male and unexplained infertility, and IVF outcome cannot be predicted on the basis of simple routine sperm analysis (Liu and Baker, 1992). This suggests the presence of a biochemical alteration of

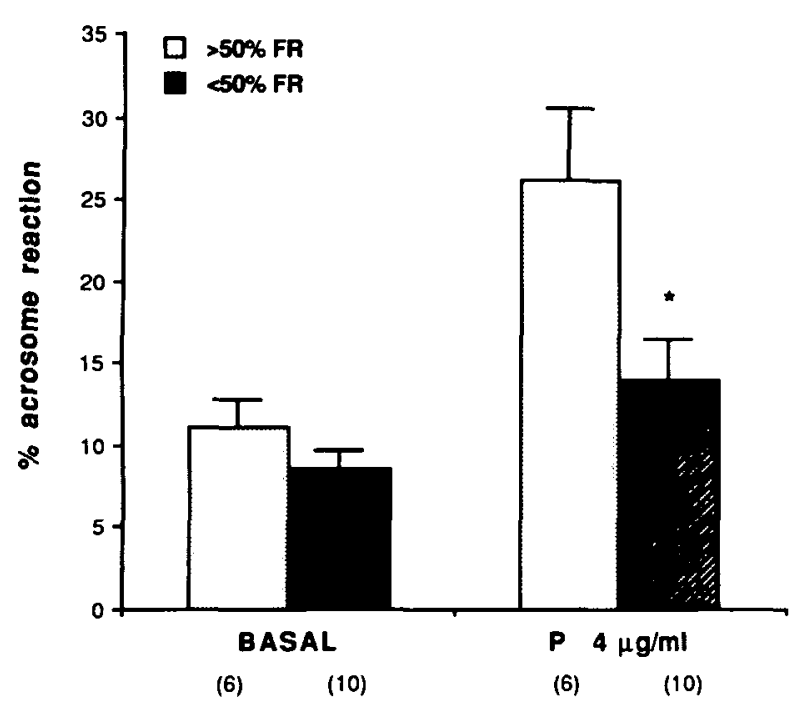

Fig. 3. Basal and progesterone (P)-stimulated acrosome reaction in spermatozoa from patients with a fertilization rate (FR) $\geq 50$ and $<50 \%$. Numbers of patients shown in parentheses. $P<0.001$ versus $\geq 50 \% \mathrm{FR}$. 
sperm function rather than defective motility or sperm concentration.

We report here that responsiveness to progesterone of spermatozoa, measured as increase of $\left[\mathrm{Ca}^{2+}\right]_{i}$ and acrosome reaction, can be of good predictive value for fertilization rate and IVF outcome. Indeed, we have shown that the $\left[\mathrm{Ca}^{2+}\right]_{i}$ as well as the acrosome reaction increases in response to progesterone were significantly higher in patients with a FR $\geq 50 \%$. Furthermore, both parameters were highly significantly

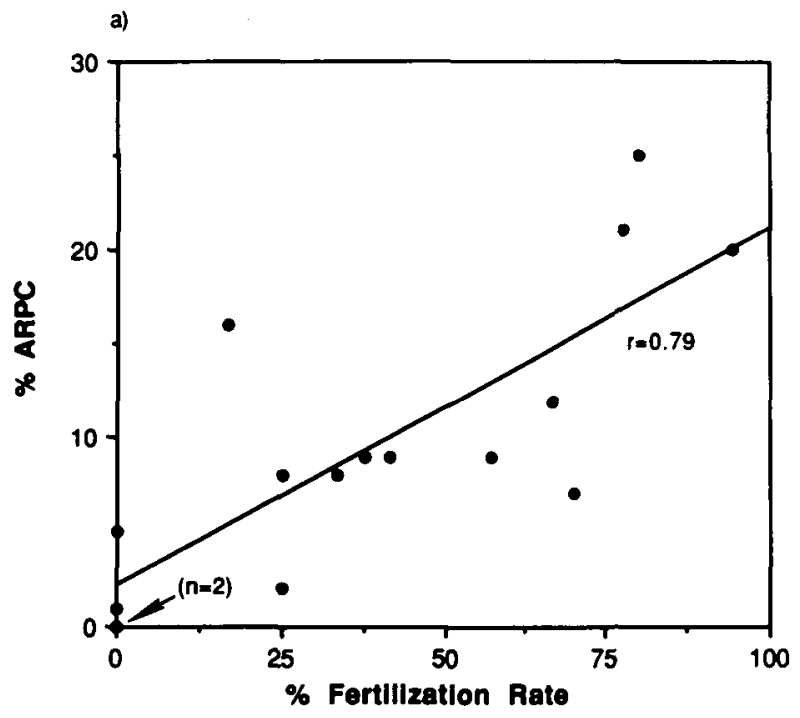

b)

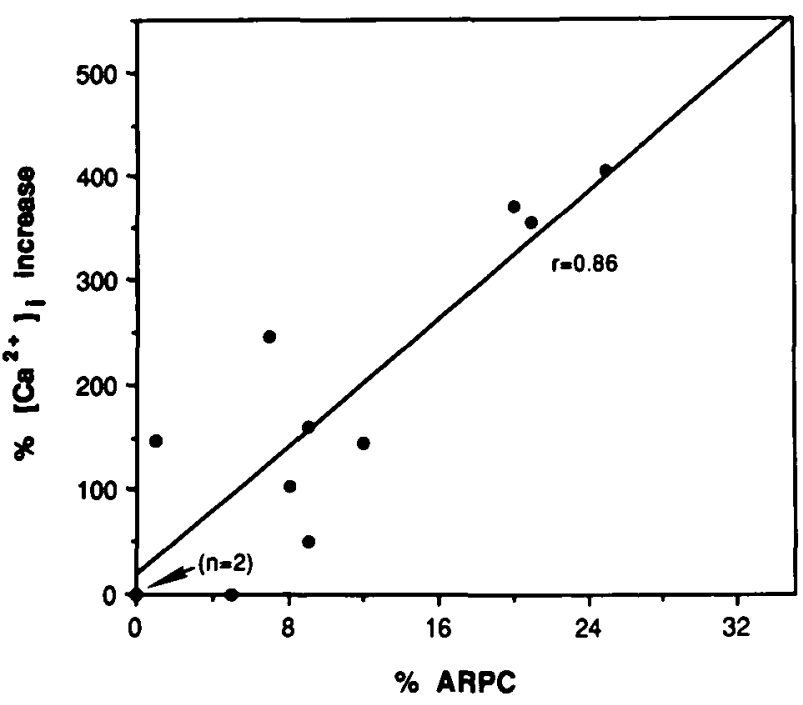

Fig. 4. (a) Correlation curve between fertilization rate and percentage acrosome reaction following progesterone challenge (ARPC) in spermatozoa from in-vitro fertilization (IVF) patients included in our study. The number of samples with zero ARPC and fertilization failure is shown in parentheses. (b) Correlation curve between percentage acrosome reaction following challenge with progesterone (ARPC) and percentage $\left[\mathrm{Ca}^{2+}\right]_{i}$ increase in response to $0.1 \mu \mathrm{g} / \mathrm{ml}$ progesterone in spermatozoa from IVF patients included in our study. The number of samples with zero ARPC and zero $\left[\mathrm{Ca}^{2+}\right]_{i}$ increase is shown in parentheses. correlated to the \%FR of the different subjects and, in cases of fertilization failure, no increase of $\left[\mathrm{Ca}^{2+}\right]_{j}$ or acrosome reaction was observed in response to progesterone (with the exception of one subject), indicating that response to progesterone is functionally related to sperm fertilizing ability. We have also shown that progesterone-stimulated $\left[\mathrm{Ca}^{2+}\right]_{i}$ and acrosome reaction increases were highly significantly correlated, indicating that the two parameters are strictly dependent and have similar predictive value for fertilization rate. On the contrary, sperm concentration and motility after swim-up preparation (and thus in the sample used for IVF) showed a poor correlation with FR, indicating that fertilizing ability cannot be predicted on the basis of these parameters.

Furthermore, although basal and post-swim-up conventional parameters were different in the two groups of subjects (Table I), of the seven patients who failed to fertilize, four were normozoospermic, further confirming that basal semen analysis has very little predictive value for fertilizing ability (Enginsu et al., 1993). Recently it has been reported that assessment of morphology with strict criteria can be predictive of IVF outcome (Enginsu et al., 1993); however, the correlation coefficient between morphology and fertilization in this study was only 0.46 . Moreover, assessment of sperm morphology is subjective, whereas progesterone-stimulated $\left[\mathrm{Ca}^{2+}\right]_{i}$ increase is an objective biochemical measurement.

Other biochemical studies have shown previously that failure of acrosomal response to calcium ionophore A23187 may be predictive of failure of oocyte fertilization (Aitken et al., 1987; Pampiglione et al., 1993) and that sperm acrosin activity is positively correlated with fertilization rate (Tummon et al., 1991; Francavilla et al., 1992; Sharma et al., 1993). The present work confirms and extends these studies to a physiological stimulus of sperm acrosome reaction and indicates that $\left[\mathrm{Ca}^{2+}\right]_{i}$ measurement following progesterone challenge can be used as an alternative to acrosome reaction in the study of sperm fertilizing ability. $\left[\mathrm{Ca}^{2+}\right]_{i}$ measurement can be easily and quickly performed in most laboratories; it utilizes readily available reagents and requires only a spectrofluorimeter equipped with a thermostat and a cuvette stirrer.

The finding of a defective response to progesterone in subjects with lower fertilizing ability is not new. A decreased response to progesterone has been shown recently in oligozoospermic men (Falsetti et al., 1993) and in some infertile patients (Tesarik and Mendoza, 1992); such studies, however, did not measure directly the correlation between response to progesterone and sperm fertilizing ability. On the other hand, Shimizu et al. (1993) reported recently a positive correlation between sperm $\left[\mathrm{Ca}^{2+}\right]_{i}$ increase in response to progesterone and hamster egg-penetrating ability in spermatozoa from fertile but not infertile men. However, the predictive value of the hamster egg penetration test versus fertilizing ability has been discussed recently ( $O$ 'Shea et al., 1993). In the present study, we directly measured the correlation between response to progesterone and the rate of fertilization of human oocytes, which was thus in a more physiological and appropriate context for the study of human sperm fertilizing ability. Moreover response to progesterone was evaluated for both increase of $\left[\mathrm{Ca}^{2+}\right]_{i}$ and acrosome reaction of spermatozoa with substantially similar results, indicating that both 
parameters can be considered a good index of sperm ability to respond to progesterone. The problem of whether sperm response to progesterone is variable from one ejaculate to another from the same subject has not been addressed in the present study and is under investigation in our laboratory. However, previous studies reporting an altered response to progesterone in oligozoospermic subjects (Falsetti et al., 1993) and in patients with a long history of infertility (Tesarik and Mendoza, 1992) suggest that this may be a permanent feature.

In conclusion, we have found that response to progesterone is a good marker of fertilizing ability of human spermatozoa which may be of predictive value for fertilization rate and IVF outcome.

\section{Acknowledgements}

We thank Prof. M.Serio (Unità di Endocrinologia, Università di Firenze) and Dr M.Maggi (Unità di Andrologia, Università di Firenze) for helpful advice. We also thank Dr S.Pratesi (Clinica Ostetrica e Ginecologica, Università di Firenze) for his clinical contribution. This work was supported by grants from Consiglio Nazionale delle Ricerche (CNR, Rome, Italy; Progetto Finalizzato FATMA, contratto 93.00097-PF41), Ministero delle Università e della Ricerca Scientifica e Tecnologica (40 and $60 \%$ funding) and Regione Toscana.

\section{References}

Aitken,R.J., Thatcher,S., Glaser,A.F., Clarckson,J.S., Wu,F.L.W. and Baird,D.T. (1987) Relative ability of modified versions of the hamster oocyte penetration test, incorporating hyperosmotic medium or the ionophore A23187, to predict IVF outcome. Hum. Reprod., 2, 227-231.

Aitken,R.J., Buckingham,D.W. and Fang,H.G. (1993) Analysis of the response of human spermatozoa to A23187 employing a novel technique for assessing acrosome reaction. J. Androl., 14, 132-141.

Baldi,E., Casano,R., Falsetti,C., Krausz,Cs., Maggi,M. and Forti,G. (1991) Intracellular calcium accumulation and responsiveness to progesterone in capacitating human spermatozoa. J. Androl., 12, $323-330$.

Enginsu,M.E., Damoulin,J.C.M., Pieters,M.H.E.C., Evers,J.L.H. and Geraedts,J.P.M. (1993) Predictive value of morphologically normal sperm concentration in the medium for in vitro fertilization. Int. J. Androl., 16, 113-120.

Falsetti,C., Baldi,E., Krausz,Cs., Casano,R., Failli,P. and Forti,G. (1993) Decreased responsiveness to progesterone of spermatozoa in oligozoospermic patients. J. Androl., 14, 17-22.

Francavilla,S., Palermo,G., Gabriele,A., Cordeschi,G. and Poccia,G. (1992) Sperm acrosin activity and fluorescence microscopic assessment of proacrosin/acrosin in ejaculates of infertile and fertile men. Fertil. Steril., 57, $1311-1316$.

Grynkiewicz,G., Poenie,M. and Tsien,R.Y. (1985) A new generation of $\mathrm{Ca}^{2+}$ indicators with greatly improved fluorescent properties. $J$. Biol. Chem., 260, 3440-3450.

Krausz,Cs., Gervasi,G., Forti,G. and Baldi,E. (1994) Effect of plateletactivating factor on motility and acrosome reaction of human spermatozoa. Hum. Reprod., 9, 471-476.

Liu,D.Y and Baker,H.W.G (1992) Tests of human sperm function and fertilization in vitro. Fertil. Steril., 58, 465-483.

Meizel,S. and Turner,K.O. (1991) Progesterone acts at the plasma membrane of human sperm. Mol. Cell. Endocrinol., 11, R1-R5.

Morales,P., Llanos,M., Gutierrez,G., Kohen,P., Vigil,P. and Vantman,D. (1992) The acrosome reaction-inducing activity of individual human follicular fluid samples is highly variable and is related to the steroid content. Hum. Reprod., 7, 646-651.
O'Shea,D.L., Odem,R.R., Cholewa,C. and Gast,M.J. (1993) Longterm follow-up of couples after hamster egg penetration testing. Fertil. Steril., 60, 1040-1045.

Osman,R.A., Andria,M.L., Jones,A.D. and Meizel,S. (1989) Steroidinduced exocytosis: the human sperm acrosome reaction. Biochem. Biophys. Res. Commun., 160, 828-833.

Pampiglione,J.S., Tan,S.-L. and Campbell,S. (1993) The use of stimulated acrosome reaction test as a test of fertilizing abilty in human spermatozoa. Fertil. Steril., 59, 1280-1284.

Saaranen,M.L., Calvo,L., Dennison,L., Banks,S., Bustillo,M., Dorfmann,A.D., Goldstein,M., Thorsell,L., Schulman,J.D. and Sherins,R.J. (1993) Acrosome reaction inducing activity in follicular fluid correlates with progesterone concentration but not with oocyte maturity or fertilizability. Hum. Reprod., 8, 1448-1454.

Sharma,R., Hogg,J. and Bromham,D.R. (1993) Is spermatozoan acrosin a predictor of fertilization and embryo quality in the human? Fertil. Steril., 60, 881-887.

Shimizu,Y., Nord,E.P. and Bronson,R.A. (1993) Progesterone-evoked increases in sperm $\left[\mathrm{Ca}^{2+}\right]_{i}$ correlate with the egg penetrating ability of sperm from fertile but not infertile men. Fertil. Steril., 60, $526-532$.

Tesarik,J. and Mendoza,C. (1992) Defective function of a non-genomic progesterone receptor as a sole sperm anomaly in infertile patients. Fertil. Steril., 58, 793-797.

Tummon,I.S., Yuzpe,A.A., Daniel,S.A.J. and Deutsch,A. (1991) Total acrosin activity correlates with fertility potential after fertilization in vitro. Fertil. Steril., 56, 933-938.

Winston,R.M. and Handyside,A.H. (1993) New challenges in human in vitro fertilization. Science, 260, 932-936.

Received on April 21, 1994; accepted on September 12, 1994 